Research Paper

\title{
Exploration of Racial Differences in Reproductive Factors for Breast Cancer among Women aged 55-74
}

\author{
Chuanbo $\mathrm{Xie}^{1 * \varpi}$, Nan Shao ${ }^{2 *}$, Longjun $\mathrm{He}^{1 *}$, Xiuhong $\mathrm{Li}^{3}$, Cong $\mathrm{Li}^{1}$, Jibin $\mathrm{Li}^{1}$, Dongfang $\mathrm{Su}^{1 凶}$ \\ 1. State Key Laboratory of Oncology in South China, Collaborative Innovation Center for Cancer Medicine, Sun Yat-sen University Cancer Center, \\ Guangzhou, China; \\ 2. Breast Disease Center, The First Affiliated Hospital of Sun Yat-Sen University, Guangzhou, Guangdong, China; \\ 3. Department of Maternal and Child Health, School of Public Health, Sun Yat-sen University, Guangzhou, Guangdong, China. \\ * Authors contributed equally to this manuscript.
}

$\square$ Corresponding authors: Chuanbo Xie, MD, PhD or Dongfang Su MD, PhD, State Key Laboratory of Oncology in South China, Collaborative Innovation Center for Cancer Medicine, Sun Yat-sen University Cancer Center, 651 Dongfengdong Road, Guangzhou 510060, China; Tel./Fax:+86-20-8734-5679; xiechb@sysucc.org.cn or sudf@sysucc.org.cn.

(c) Ivyspring International Publisher. This is an open access article distributed under the terms of the Creative Commons Attribution (CC BY-NC) license (https://creativecommons.org/licenses/by-nc/4.0/). See http://ivyspring.com/terms for full terms and conditions.

Received: 2017.09.18; Accepted: 2017.11.03; Published: 2018.01.01

\begin{abstract}
Background Reproductive factors have been well-documented risk factors for breast cancer. Few studies have examined whether the associations between reproductive factors and breast cancer differed across races/ethnicities. Methods We analyzed a sub-sample $(70,734)$ of the Prostate, Lung, Colorectal, and Ovarian (PLCO) dataset. Participants with valid baseline questionnaire and without breast cancer at enrollment were included into analysis. We stratified the participants into subgroups based on their races/ethnicities then estimated the effects of the reproductive factors on breast cancer within each group using Cox-proportion regression models. Results Oral contraceptive use $(\mathrm{HR}=1.09,95 \%$ confidence interval or $\mathrm{Cl}=1.01,1.18)$, advanced age at natural menopause $(\mathrm{HR}=1.25,95 \% \mathrm{Cl}=1.06,1.49)$ were associated with increased risk of breast cancer in non-Hispanic Caucasians group only. Long term use of menopausal hormone therapy (more than five years) was associated with increased risk of breast cancer in both of the non-Hispanic Caucasian $(H R=1.44,95 \% \mathrm{Cl}=1.31,1.59)$ group and the non-Hispanic Asian/Pacific Islander $(H R=1.98,95 \%$ $\mathrm{Cl}=1.23,3.20)$ group, but not in other race/ethnic groups. Hispanics who tried to become pregnant for a year or more had increased risk of breast cancer $(H R=2.60,95 \% \mathrm{Cl}=1.05,6.46)$ than their counterparts without difficulty in getting pregnancy. In addition, surgery induced menopause was found to be a protective factor for breast cancer in non-Hispanic Caucasian $(\mathrm{HR}=0.88,95 \% \mathrm{Cl}=0.79$, 0.98) group only.

Conclusions We concluded that different races/ethnicities had different breast cancer related reproductive risk factors. Non-Hispanic Caucasians had the most breast cancer related reproductive risk factors, while the minorities had none or few breast cancer related reproductive risk factors and among these few factors only 1 was also risk factor for non-Hispanic Caucasians.
\end{abstract}

Key words: breast cancer, reproductive factors, race differences, Prostate, Lung, Colorectal and Ovarian (PLCO) Cancer Screening Trial.

\section{Introduction}

Breast cancer incidences vary greatly across races/ethnicities, with high incidences in non-Hispanic Caucasians and African-Americans and low incidences in Asians and Hispanics whose age are more than 60 years ${ }^{1}$. According to the annual cancer status report (1975-2014), the age standardized incidences of breast cancer in the United States for non-Hispanic Caucasians, African-Americans, Asian/ Pacific Islanders, and Hispanics during the period 2009 to 2013 were 126.9, 125.3, 93.4, and 95.6 per 100 000, respectively 2. Although variation in social economic status (SES), screening frequencies, cancer 
reporting practices, and prevalence of risk factors might partially explain the disparities of breast cancer incidences across races/ethnicities, we suspected that the varied effects of breast cancer risk factors among different races/ethnicities might also contribute to these differences.

Reproductive factors such as advanced age at pregnancy, early age at menarche, and advanced age at menopause are well-documented risk factors for breast cancer ${ }^{5,6}$ and some of them are routinely used to predict woman's breast cancer risk (e.g., Gail model $)^{7}$. However, different races/ethnicities have differential genetic backgrounds, breast tissue density, and estrogen sensitivities, thus the effects of these reproductive risk factors on breast cancer are very likely different across races/ethnicities. Until now, only few studies have tested this hypothesis ${ }^{8-10}$. Unfortunately, of these studies, the researchers compared the effects of reproductive factors on breast cancer only in limited race groups and did not include non-Hispanic Asian/Pacific Islanders whose population size is increasing rapidly in United States $8-10$.

In this study, we aimed to determine whether the effects of some specific reproductive risk factors on breast cancer varied across races/ethnicities by analyzing a subset of Prostate, Lung, Colorectal, and Ovarian (PLCO) study dataset in which the non-Hispanic Asian/Pacific Islanders accounted for about $4.0 \%$. Our study could shed light on screening racial/ethnic specific breast cancer related reproductive risk factors and explaining the disparities in breast cancer incidences across different races/ethnicities.

\section{Materials and Methods}

\section{Setting and Data Collection}

We analyzed a sub-sample of the Prostate, Lung, Colorectal and Ovarian (PLCO) Cancer Screening dataset. The detailed design of PLCO study can be found elsewhere ${ }^{12}$. Briefly, PLCO study is a randomized clinical trial aiming at determining whether the traditional screening tests (PSA blood tests, digital rectal exams, chest $\mathrm{X}$-ray, flexible sigmoidoscopy, cancer antigen 125, and transvaginal ultrasound) reduce the mortality from prostate, lung, colorectal, and ovarian cancers. This trial was conducted in ten centers across U.S. (i.e., Alabama, Michigan, Colorado, Hawaii, Wisconsin, Minnesota, Pennsylvania, Utah, Missouri, and Washington DC). Nine centers started recruitment in 1993 and one center in 1998. All the centers ended recruitment at 2001. The eligibility criteria for participants were listed as below: age between 55-74 years, with no previous history of prostate, lung, colorectal, or ovarian cancer, and not participate in other cancer screening or prevention trials. After entry, the participants were randomly assigned into intervention arm or control arm. The participants in the intervention arm received series of screening tests for prostate, lung, colorectal, and ovarian cancers. The participants in control arm only received routine health care from their health care providers. The screening process for the intervention group ended in 2006, but the annual followup continues for more than ten years (i.e., median followup time $=12.5$ years). All the participants signed the study informed consent forms which had been reviewed and approved by the Institutional Review Board at the National Cancer Institute and local sites.

\section{Participants}

In this study, participants with valid baseline questionnaire and completed breast cancer information in both intervention and control arms were considered as eligible. Figure $\mathbf{1}$ shows the analytic sample selection process. Briefly, a total of 154,897 participants were enrolled into PLCO study at baseline and among them 78,215 were females. Among the female participants, 7,273 were diagnosed as cancer patients at baseline and thus were excluded from our analysis. Finally, 70,942 female participants (i.e., 35,550 in the intervention arm and 35,392 in the control arm) without cancer history at baseline were included into analysis.

\section{Exposure}

At baseline, $96.8 \%$ of the participants completed a self-reported questionnaire to collect demographics, smoking history, family history of cancer, body mass index (BMI), medical conditions, personal history of cancer, and reproductive history information. We defined reproductive history information as the main exposure of this study. Reproductive information included oral contraceptive (OC) use (i.e., Yes vs. No), menopausal hormone therapy (MHT) use (i.e., never, former, current user less than 5 years, and current users more than 5 years), age at birth of first child (i.e., less than 20, 20-24, 25-29, 30-34, and $\geq 35$ years ) , age at first pregnancy $(\leq 19,20-24,25-29,30-34$, and $\geq 35$ years), numbers of early termination of pregnancies (none, one, two or more), numbers of pregnancies (none, one, two, three to four, five to nine, and more than ten), age at menarche (i.e., $\leq 11,12-13,14-15, \geq 16$ years), age at natural menopause (i.e., <40, 40-44, $45-49,50-54, \geq 55$ years), and menopause type (i.e., natural menopause, radiation and drug therapy induced menopause, surgery induced menopause). 


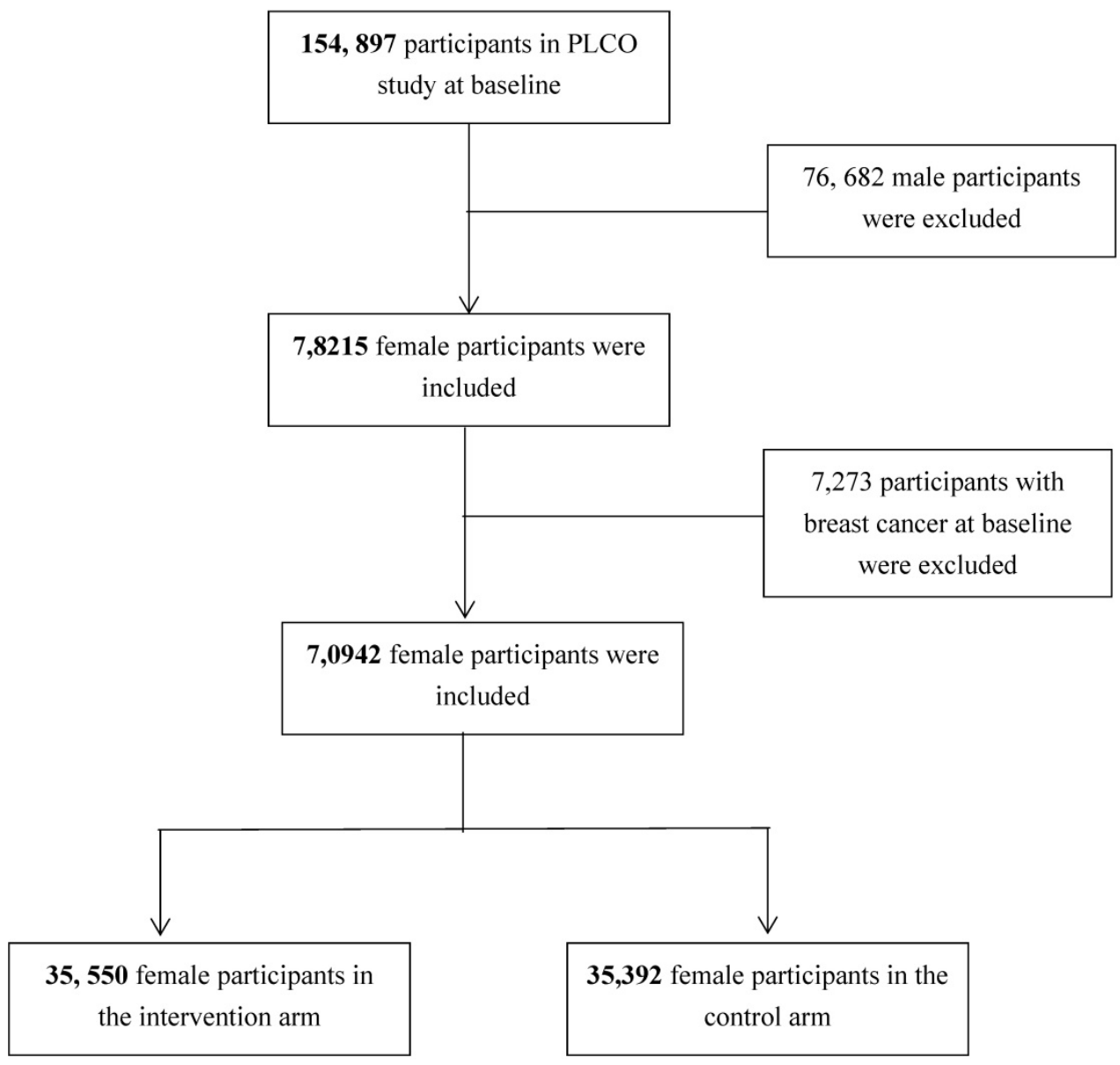

Figure 1. Flow chart of the analytic sample selection process.

\section{Outcomes}

The outcome of this analysis is the incidence of breast cancer post study enrollment. Researchers collected breast cancer information by mailing paper based self-reported annual study update (ASU) questionnaire, giving telephone calls, reviewing medical records and/or death certificate. In more recent years, state cancer registries were used to identify/confirm breast cancer diagnosis. We defined the follow-up time (in days) as the time interval between the date from trial entry (randomization) to the date of breast cancer diagnosis, death, or last contact. We defined observations with breast cancer diagnosis as complete data and observations with death or losing contact as censored data.

\section{Covariates}

According to our experience and the literature, we considered age at baseline, educational level, marital status, family female breast cancer history, current BMI status, benign breast diseases history, and all the other reproductive factors as confounding factors. The PLCO researchers collected the confounding factors information via the baseline questionnaire. Age at baseline was included into analysis as a continuous variable, while other variables were included as categorical variables.

\section{Statistical analysis}

We used mean and standard deviation (SD) or median and interquartile range (IQR) to describe continuous variables and percentages to describe categorical variables. We estimated the overall (i.e., all races/ethnicities) hazard ratios (HR) and 95\% confidence intervals $(\mathrm{CI})$ of each reproductive factor on breast cancer using Cox proportional hazards models. Then we stratified the participants into different race groups and estimated the effects of reproductive factors on breast cancer (HR and 95\% CI) within each group using multiple Cox proportional hazards regression models. Finally, we estimate the differences in effects of breast cancer of each reproductive factor across races/ethnicities by adding the interaction terms of "reproductive factor $\times$ races/ethnicities" into Cox regression models. All the data analyses were performed by SAS 9.3 software (SAS Institute, Cary, North Carolina, USA).The significant level a was set as 0.05 . 


\section{Results}

\section{Sample characteristics}

Table 1 shows the characteristics of the included participants. The mean age of the included participants was 62 years $(S D=5.4)$. Non-Hispanic African American group has the lowest married status $(39.6 \%)$, family female breast cancer $(11.6 \%)$, and MHT use (49.9\%), but the highest obesity rate (45.5\%). Non-Hispanic Asian/Pacific Islanders had the highest educational level (36.1\% with graduate or above), never smoking rate $(69.0 \%)$, and benign breast diseases $(15.8 \%)$, but the lowest obesity rate $(9.2 \%)$. Hispanics had the lowest educational level (19.1\% with eleventh grade or below). The median follow up time of the included participants was 4203 days (11.5 years) and a total of 4054 patients (5.7\%) developed breast cancer during the study period.

\section{Reproductive factors and breast cancer for all races/ethnicities combined}

Table 2 shows the associations between reproductive factors and breast cancer. Compared to women who never used MHT, current MHT users had significantly higher risk of breast cancer (adjusted HRs for $<5$ year user and for $\geq 5$ year users were 1.24 (95\% CI=1.10, 1.37) and 1.44 (95\% CI=1.31, 1.58), respectively). Women with age at first pregnancy more than 35 years had significant higher risk of breast cancer $(\mathrm{HR}=1.40,95 \% \mathrm{CI}=1.12,1.75)$ than women with age at first pregnancy less than 19 years. Our crude data analysis suggested that breast cancer risks gradually reduced with numbers of pregnancies increasing, however this association disappeared after adjusting for potential confounding factors. Compared to women with age at natural menopause less than 40 years, women with natural menopause age between $50-54$ years $(\mathrm{HR}=1.15,95 \% \mathrm{CI}=1.00,1.33)$ and with age more than 55 years $(\mathrm{HR}=1.24,95 \%$ $\mathrm{CI}=1.06,1.46)$ were more likely to have breast cancer. In addition, we found that surgery induced menopausal was associated with reduced breast cancer risk $(\mathrm{HR}=0.88,95 \% \mathrm{CI}=0.82,0.94)$.

Table 1. Characteristics of the included participants.

\begin{tabular}{|c|c|c|c|c|c|c|}
\hline Characteristics & $\begin{array}{l}\text { All a } \\
(N=70,734)\end{array}$ & $\begin{array}{l}\text { non-Hispanic } \\
\text { Caucasians a } \\
(n=62,717)\end{array}$ & $\begin{array}{l}\text { non-Hispanic } \\
\text { African Americans a } \\
(n=4,099)\end{array}$ & $\begin{array}{l}\text { non-Hispanic } \\
\text { Asian/Pacific Islander a } \\
(\mathrm{n}=2,781)\end{array}$ & $\begin{array}{l}\text { Hispanics a } \\
(n=1,137)\end{array}$ & $P$-value ${ }^{b}$ \\
\hline Age in years, mean (SD) & $62(5.4)$ & $62(5.4)$ & $62(5.5)$ & $63(5.5)$ & $62(5.1)$ & $<0.001$ \\
\hline \multicolumn{7}{|l|}{ Educational level, n (\%) } \\
\hline Eleven grade or below & $4584(6.5)$ & $3512(5.6)$ & $681(16.7)$ & $175(6.4)$ & $216(19.1)$ & \multirow[t]{4}{*}{$<0.001$} \\
\hline High school & $19561(27.7)$ & $17752(28.4)$ & $859(21.0)$ & $682(24.8)$ & $268(23.7)$ & \\
\hline College or equal & $25390(36.0)$ & $22662(36.2)$ & 1455 (35.6) & $901(32.7)$ & $372(32.9)$ & \\
\hline Graduate or above & $21026(29.8)$ & $18663(29.8)$ & $1094(26.8)$ & $994(36.1)$ & $275(24.3)$ & \\
\hline \multicolumn{7}{|l|}{ Marital status, $\mathrm{n}(\%)$} \\
\hline Married or living as married & $48840(69.2)$ & $44633(71.3)$ & $1620(39.6)$ & $1913(69.4)$ & $674(59.7)$ & \multirow[t]{4}{*}{$<0.001$} \\
\hline Widowed & $9716(13.8)$ & $8168(13.1)$ & $980(24.0)$ & 410 (14.9) & $158(14.0)$ & \\
\hline Divorced/separated & 9660 (13.7) & $7827(12.5)$ & 1265 (30.9) & $313(11.4)$ & 255 (22.6) & \\
\hline Never married & $2364(3.4)$ & $1976(3.2)$ & $225(5.5)$ & $120(4.4)$ & $43(3.8)$ & \\
\hline \multicolumn{7}{|l|}{ Current smoking status, $\mathrm{n}(\%)$} \\
\hline Never smoker & $39656(56.1)$ & 35061 (55.9) & $2058(50.2)$ & $1917(69.0)$ & $620(54.6)$ & \multirow[t]{3}{*}{$<0.001$} \\
\hline Current smoker & $6765(9.6)$ & $5829(9.3)$ & $632(15.4)$ & $185(6.7)$ & $119(10.5)$ & \\
\hline Former smoker & $24307(34.4)$ & $21823(34.8)$ & $1409(34.4)$ & $678(24.4)$ & $397(35.0)$ & \\
\hline \multicolumn{7}{|l|}{ Family female breast cancer, $\mathrm{n}(\%)$} \\
\hline No & $59809(86.0)$ & $52933(85.8)$ & $3533(88.4)$ & $2389(87.6)$ & $954(85.9)$ & \multirow[t]{2}{*}{$<0.001$} \\
\hline Yes & $9710(14.0)$ & $8754(14.2)$ & $462(11.6)$ & $337(12.4)$ & $157(14.1)$ & \\
\hline \multicolumn{7}{|l|}{ Current $\mathrm{BMI}$ in $\mathrm{kg} / \mathrm{m}^{2}, \mathrm{n}(\%)$} \\
\hline $0-18.5$ & $776(1.1)$ & $650(1.1)$ & $22(0.6)$ & $91(3.4)$ & $13(1.2)$ & \multirow[t]{4}{*}{$<0.001$} \\
\hline $18.5-25$ & $27420(39.3)$ & 24718 (39.9) & $714(17.9)$ & $1610(59.3)$ & $378(34.4)$ & \\
\hline $25-30$ & $24198(34.7)$ & $21622(34.9)$ & $1434(36.0)$ & $762(28.1)$ & $380(34.6)$ & \\
\hline$>30$ & $17330(24.9)$ & $14942(24.1)$ & $1811(45.5)$ & $250(9.2)$ & $327(29.8)$ & \\
\hline \multicolumn{7}{|l|}{ Benign breast diseases, $\mathrm{n}(\%)$} \\
\hline No & $49740(72.1)$ & $43570(70.9)$ & $3106(81.4)$ & $2225(84.2)$ & $839(76.1)$ & \multirow[t]{2}{*}{$<0.001$} \\
\hline Yes & $19276(27.9)$ & $17883(29.1)$ & $711(18.6)$ & $418(15.8)$ & $264(23.9)$ & \\
\hline \multicolumn{7}{|l|}{ Menopausal hormone therapy use, n (\%) } \\
\hline Never & $23092(32.9)$ & $19806(31.8)$ & $2024(50.1)$ & $863(31.7)$ & $399(35.4)$ & \multirow[t]{4}{*}{$<0.001$} \\
\hline Former user & $11493(16.4)$ & $10073(16.2)$ & $807(20.0)$ & $453(16.6)$ & $160(14.2)$ & \\
\hline Current user less than 5 years & $11722(16.7)$ & $10453(16.8)$ & $537(13.3)$ & $549(20.2)$ & $183(16.3)$ & \\
\hline Current user more than 5 years & $23842(34.0)$ & $21928(35.2)$ & $672(16.6)$ & $858(31.5)$ & $384(34.1)$ & \\
\hline Breast cancer, n (\%) & $4054(5.7)$ & $3665(5.8)$ & $169(4.1)$ & $164(5.9)$ & $56(4.9)$ & $<0.001$ \\
\hline Follow up time in days, median (IQR) & $(966)$ & $(900)$ & $(1471)$ & $(868)$ & $(1110)$ & \multirow[t]{2}{*}{$<0.001$} \\
\hline [Follow up time in years, medians (IQR)] & {$[11.5(2.6)]$} & {$[11.5(2.5)]$} & {$[10.5(4.0)]$} & {$[12.6(2.4)]$} & {$[10.8(3.0)]$} & \\
\hline
\end{tabular}


Table 2. Associations between reproductive factors and breast cancer in PLCO study.

\begin{tabular}{|c|c|c|c|c|c|c|}
\hline \multirow[t]{2}{*}{ Reproductive factors } & \multirow[t]{2}{*}{$\mathrm{N},(\%)$} & \multicolumn{3}{|l|}{ Breast cancer } & \multicolumn{2}{|l|}{ Breast cancer } \\
\hline & & $\%$ developed breast cancer & Crude HR $(95 \% \mathrm{CI})$ & $P$-value & Adjusted HR ${ }^{\mathrm{b}}(95 \% \mathrm{CI})$ & $P$-value \\
\hline \multicolumn{7}{|l|}{ Ever take oral contraceptive pills } \\
\hline No & $32237(45.7)$ & 5.7 & Ref & & Ref & \\
\hline Yes & $38374(54.4)$ & 5.8 & $1.03(0.97,1.10)$ & 0.304 & $1.07(0.99,1.15)$ & 0.082 \\
\hline \multicolumn{7}{|l|}{ Menopausal hormone therapy use } \\
\hline Never & $23092(32.9)$ & 4.9 & Ref & & Ref & \\
\hline Former user & $11493(16.4)$ & 5.2 & $1.05(0.95,1.16)$ & 0.351 & $1.06(0.95,1.19)$ & 0.275 \\
\hline Current user less than 5 years & $11722(16.7)$ & 6.2 & $1.24(1.13,1.37)$ & $<0.001$ & $1.22(1.10,1.37)$ & 0.000 \\
\hline Current user more than 5 years & $23842(34.0)$ & 6.6 & $1.35(1.25,1.46)$ & $<0.001$ & $1.44(1.31,1.58)$ & $<0.001$ \\
\hline \multicolumn{7}{|l|}{ Age at birth of first child } \\
\hline$\leq 19$ & $12052(18.9)$ & 5.0 & Ref & & Ref & \\
\hline $20-24$ & $32817(51.3)$ & 5.4 & $1.07(0.97,1.17)$ & 0.182 & $0.86(0.72,1.01)$ & 0.072 \\
\hline $25-29$ & $14165(22.2)$ & 6.1 & $1.19(1.07,1.32)$ & 0.001 & $0.88(0.70,1.10)$ & 0.259 \\
\hline $30-34$ & $3650(5.7)$ & 7.1 & $1.40(1.21,1.62)$ & $<0.001$ & $1.05(0.78,1.40)$ & 0.766 \\
\hline$\geq 35$ & $1239(1.9)$ & 7.0 & $1.40(1.12,1.75)$ & 0.003 & $0.84(0.55,1.29)$ & 0.433 \\
\hline \multicolumn{7}{|c|}{ Numbers of early termination of pregnancy } \\
\hline None & $46377(65.8)$ & 5.8 & Ref & & Ref & \\
\hline One & $15539(22.0)$ & 5.6 & $0.97(0.90,1.05)$ & 0.507 & $1.03(0.95,1.13)$ & 0.485 \\
\hline Two or more & 8579 (12.2) & 5.8 & $1.01(0.92,1.11)$ & 0.885 & $1.12(0.99,1.26)$ & 0.080 \\
\hline \multicolumn{7}{|l|}{ Ever been pregnant } \\
\hline No & $5218(7.4)$ & 7.0 & Ref & & Ref & \\
\hline Yes & $65421(92.6)$ & 5.6 & $0.79(0.71,0.88)$ & $<0.001$ & - & \\
\hline \multicolumn{7}{|c|}{ Age in years when first became pregnant } \\
\hline$\leq 19$ & $16472(25.2)$ & 4.9 & Ref & & Ref & \\
\hline $20-24$ & $32608(49.9)$ & 5.6 & $1.13(1.04,1.23)$ & 0.005 & $1.19(1.02,1.38)$ & 0.031 \\
\hline $25-29$ & $12490(19.1)$ & 6.2 & $1.25(1.13,1.38)$ & $<0.001$ & $1.19(0.96,1.48)$ & 0.114 \\
\hline $30-34$ & $2841(4.4)$ & 6.9 & $1.40(1.20,1.64)$ & $<0.001$ & $1.14(0.83,1.56)$ & 0.415 \\
\hline$\geq 35$ & $894(1.4)$ & 8.2 & $1.68(1.32,2.14)$ & $<0.001$ & $1.68(1.03,2.77)$ & 0.039 \\
\hline \multicolumn{7}{|l|}{ Number of pregnancies } \\
\hline None & $5218(7.4)$ & 7.0 & Ref & & Ref & \\
\hline One & $4036(5.7)$ & 6.1 & $0.88(0.75,1.04)$ & 0.130 & - & \\
\hline Two & $12481(17.7)$ & 6.0 & $0.85(0.75,0.96)$ & 0.010 & $0.95(0.81,1.12)$ & 0.539 \\
\hline Three to four & $28652(40.6)$ & 5.7 & $0.79(0.71,0.89)$ & $<0.001$ & $0.93(0.79,1.10)$ & 0.399 \\
\hline Five to nine & $18800(26.6)$ & 5.3 & $0.73(0.65,0.83)$ & $<0.001$ & $0.88(0.74,1.05)$ & 0.166 \\
\hline More than ten & $1391(2.0)$ & 4.5 & $0.62(0.47,0.81)$ & 0.001 & $0.73(0.53,1.01)$ & 0.055 \\
\hline \multicolumn{7}{|c|}{ Ever tried to become pregnant for a year or more } \\
\hline No & $60284(85.6)$ & 5.6 & Ref & & Ref & \\
\hline Yes & $10140(14.4)$ & 6.4 & $1.14(1.05,1.24)$ & 0.002 & $1.08(0.98,1.20)$ & 0.124 \\
\hline \multicolumn{7}{|l|}{ Age at menarche } \\
\hline$\leq 11$ years & $14294(20.3)$ & 6.1 & Ref & & Ref & \\
\hline $12-13$ years & $37922(53.8)$ & 5.7 & $0.92(0.85,1.00)$ & 0.048 & $0.92(0.84,1.00)$ & 0.058 \\
\hline $14-15$ years & $15107(21.4)$ & 5.6 & $0.90(0.82,0.99)$ & 0.031 & $0.91(0.82,1.02)$ & 0.093 \\
\hline$\geq 16$ years & $3219(4.6)$ & 5.3 & $0.87(0.74,1.03)$ & 0.096 & $0.86(0.72,1.04)$ & 0.120 \\
\hline \multicolumn{7}{|l|}{ Age at menopause } \\
\hline$<40$ & 9727 (13.9) & 4.8 & Ref & & Ref & \\
\hline $40-44$ & $9880(14.1)$ & 5.3 & $1.12(0.99,1.27)$ & 0.079 & $1.08(0.94,1.25)$ & 0.262 \\
\hline $45-49$ & $16733(23.9)$ & 5.4 & $1.13(1.01,1.26)$ & 0.034 & $1.06(0.93,1.21)$ & 0.405 \\
\hline $50-54$ & $25831(36.8)$ & 6.0 & $1.24(1.12,1.38)$ & $<0.001$ & $1.15(1.00,1.33)$ & 0.043 \\
\hline$\geq 55$ & 7955 (11.3) & 7.0 & $1.44(1.27,1.63)$ & $<0.001$ & $1.24(1.06,1.46)$ & 0.009 \\
\hline \multicolumn{7}{|l|}{ Menopausal type } \\
\hline Natural Menopause & $43703(63.0)$ & 5.9 & Ref & & Ref & \\
\hline Radiation/Drug Therapy & $23329(33.6)$ & 5.1 & $1.38(1.19,1.60)$ & $<0.001$ & $1.15(0.97,1.36)$ & 0.119 \\
\hline Surgery & $2355(3.4)$ & 8.0 & $0.88(0.82,0.94)$ & $<0.001$ & $0.86(0.78,0.95)$ & 0.004 \\
\hline
\end{tabular}

\section{Reproductive factors and breast cancer stratified by races/ethnicities}

Table 3 shows the incidences of breast cancer among each reproductive factor and race/ethnicity categorized group. Table 4 shows the associations between reproductive factors and breast cancer for each race/ethnicity group after adjusting for potential confounding factors. We found that oral contraceptive
(OC) use was associated with increased risk of breast cancer in non-Hispanic Caucasian group (HR=1.09, $95 \% \mathrm{CI}=1.01,1.18$ ) but not in other race groups. Our interaction analysis showed that the effects of oral contraceptive use on breast cancer significantly differed across races/ethnicities $(\mathrm{P}=0.047)$. Compared to their counterparts with natural menopause, non-Hispanic Caucasians $(\mathrm{HR}=0.88,95 \% \mathrm{CI}=0.79$, $0.98)$ and non-Hispanic African-Americans $(\mathrm{HR}=0.62$, 
95\% CI $=0.38,1.01)$ who had surgery induced menopause tended to have reduced risk of breast cancer, however non-Hispanic Caucasian with radiation/drug induced menopause had marginally increased risk of breast cancer $(\mathrm{HR}=1.17,95 \% \mathrm{CI}=0.99$, 1.39). Interaction analysis suggested that the effects of types of menopause on breast cancer significantly differed across races/ethnicities $(\mathrm{P}=0.026)$. We also found that long term MHT use (i.e., more than 5 years) was associated with increased risk of breast cancer in non-Hispanic Caucasians (HR=1.44, 95\% CI=1.31, 1.59) and non-Hispanic Asian/Pacific Islanders $(\mathrm{HR}=1.98,95 \% \mathrm{CI}=1.23,3.20)$ but not in other races/ethnicities. Non-Hispanic Caucasians with first pregnancy age between 20-24 years had increased risk of breast cancer than their counterparts with age less than 19 years $(\mathrm{HR}=1.20,95 \% \mathrm{CI}=1.02,1.41)$. Hispanics who ever tried to become pregnant for more than 1 year had significantly increased risk of breast cancer $(\mathrm{HR}=2.60,95 \% \mathrm{CI}=1.05,6.46)$ than their counterparts without difficulties in getting pregnancy. Non-Hispanic Caucasians with age at natural menopause greater than 55 years $(\mathrm{HR}=1.25,95 \%$ $\mathrm{CI}=1.06,1.49$ ) had significantly higher risk of breast cancer than women with age at natural menopause less than 40 years.

\section{Discussion}

In this subset of PLCO screening data sample, we found that different races/ethnicities had different breast cancer related reproductive risk factors. In non-Hispanic Caucasians, OC use, younger age at first pregnancy (i.e. 20-24 years), menopausal hormone therapy use, and advanced age at natural menopause were associated with increased risk of breast cancer, while more than ten times of pregnancies and surgery induced menopause reduced breast cancer risk. However, none of the reproductive factors were associated with breast cancer among non-Hispanic African Americans. Similar with non-Hispanic Caucasians, non-Hispanic Asian/Pacific Islanders who used menopausal hormone therapy for more than 5 years had increased risk of breast cancer. In Hispanics, we found that women who ever tried to get pregnant for more than one year had significantly increased risk of breast cancer.

In line with the literature ${ }^{13}$, we found that OC use was associated with borderline increased risk of breast cancer, which might be explained by the breast cell proliferation effect of $\mathrm{OC}^{14}$. However, our stratification analysis suggested that OC use was associated with increased risk of breast cancer in
non-Hispanic Caucasian group only but not in other race groups. We speculate that this phenomenon could be explained by the differences in timing, frequencies, and duration of OC use. Compared to other races/ethnicities, non-Hispanic Caucasians were more likely to use OC before first full-term birth $^{15}$ and with longer duration ${ }^{15}$, both of which were well-documented risk factor for breast cancer. Although only with slightly increased risk of breast cancer after OC use, non-Hispanic Caucasians seemed likely to be breast cancer venerable after OC use and they were expected to balance the pros and cons when considering to use OC especially before first full term birth.

Menopause hormone replacement therapy (MHT) was widely used for relief of menopausal symptoms such as hot flashes, sleep disturbances, vaginal dryness, and osteoporosis ${ }^{17}$. Although observational studies showed that MHT was associated with increased risk of breast cancer, the clinicians started to prescribe much lower doses of hormone for much shorter terms since 2002 when the Women's Health Initiative (WHI) study first confirmed that MHT use was associated with increased risk of breast cancer using a randomized clinical trial design ${ }^{17}$. In line with the literature, we found that MHT use was associated with increased risk of breast cancer in non-Hispanic Caucasian and non-Hispanic Asian/Pacific Islander groups but not in non-Hispanic African-American and Hispanic groups; and the effect of MHT on breast cancer was slightly stronger in non-Hispanic Asian/Pacific Islanders than that in non-Hispanic Caucasians ${ }^{19}$. This may be explained by the differed breast densities, MHT prescribing patterns, and estrogen sensitivities across different races/ethnicities. First, the breast densities among non-Hispanic Caucasians could be significantly higher than that of non-Hispanic African Americans but lower than that of non-Hispanic Asian/Pacific Islanders ${ }^{19}$. Therefore, the effects of exogenous hormones may be moderated by breast cancer densities across races/ethnicities. Second, compared with non-Hispanic Caucasians and Asians, non-Hispanic African Americans might less likely to prescribe combined estrogen and progestin ${ }^{21}$, which had stronger breast cancer effect than estrogen only ${ }^{22}$. Finally, previous studies suggested that Asian women had lower estrogen levels than their non-Hispanic Caucasian counterparts ${ }^{23}$. Therefore, it is possible that Asians are more sensitive to exogenous source of circulating hormones than non-Hispanic Caucasians and thus are at higher risk of breast cancer at the same exposure level. 
Table 3. Breast cancer incidences of the participants stratified by reproductive characteristics and races/ethnicities in the PLCO study.

\begin{tabular}{|c|c|c|c|c|c|c|c|c|}
\hline \multirow[t]{2}{*}{ Reproductive factors } & \multicolumn{2}{|c|}{$\begin{array}{l}\text { non-Hispanic Caucasians a } \\
(n=62,717)\end{array}$} & \multicolumn{2}{|c|}{$\begin{array}{l}\text { non-Hispanic } \\
\text { African Americans a }(n=4,099)\end{array}$} & \multicolumn{2}{|c|}{$\begin{array}{l}\text { non-Hispanic Asian/Pacific } \\
\text { Islander a }(n=2,781)\end{array}$} & \multicolumn{2}{|l|}{$\begin{array}{l}\text { Hispanics a } \\
(n=1,137)\end{array}$} \\
\hline & n (\%) & $\begin{array}{l}\% \text { developed } \\
\text { breast cancer }\end{array}$ & n (\%) & $\begin{array}{l}\% \text { developed } \\
\text { breast cancer }\end{array}$ & n (\%) & $\begin{array}{l}\% \text { developed } \\
\text { breast cancer }\end{array}$ & n (\%) & $\begin{array}{c}\% \text { developed } \\
\text { breast cancer }\end{array}$ \\
\hline \multicolumn{9}{|l|}{ Ever take oral contraceptive pills } \\
\hline No & $28167(44.9)$ & 5.7 & $538(47.3)$ & 4.6 & $1707(61.4)$ & 6.1 & $538(47.3)$ & 5.2 \\
\hline Yes & $34478(55.0)$ & 6.0 & $594(52.2)$ & 3.8 & $1044(37.5)$ & 5.7 & $594(52.2)$ & 4.7 \\
\hline Missing & $72(0.1)$ & & $5(0.4)$ & & $30(1.1)$ & & $5(0.4)$ & \\
\hline \multicolumn{9}{|l|}{$\begin{array}{l}\text { Menopausal hormone therapy } \\
\text { treatment } \mathrm{b}\end{array}$} \\
\hline Never & $19806(31.6)$ & 5.0 & $399(35.1)$ & 4.6 & $863(31.0)$ & 4.5 & $399(35.1)$ & 4.8 \\
\hline Former user & $10073(16.1)$ & 5.3 & $160(14.1)$ & 3.2 & $453(16.3)$ & 6.0 & $160(14.1)$ & 4.4 \\
\hline Current user less than 5 years & $10453(16.7)$ & 6.2 & $183(16.1)$ & 3.7 & 549 (19.7) & 6.4 & $183(16.1)$ & 8.7 \\
\hline Current user more than 5 years & $21928(35.0)$ & 6.7 & $384(33.8)$ & 3.9 & $858(30.9)$ & 7.2 & $384(33.8)$ & 3.7 \\
\hline Missing & $457(0.7)$ & & $11(1.0)$ & & $58(2.1)$ & & $11(1.0)$ & \\
\hline \multicolumn{9}{|l|}{ Age at birth of first child ${ }^{c}$} \\
\hline Less than 20 years & $10067(16.1)$ & 5.0 & $290(25.5)$ & 4.8 & $244(8.8)$ & 3.3 & $290(25.5)$ & 5.2 \\
\hline 20-24 years & 29915 (47.7) & 5.5 & $489(43.0)$ & 3.6 & $988(35.5)$ & 5.7 & $489(43.0)$ & 4.7 \\
\hline 25-29 years & $12616(20.1)$ & 6.3 & $167(14.7)$ & 3.3 & $862(31.0)$ & 5.2 & $167(14.7)$ & 3.0 \\
\hline 30-34 years & $3177(5.1)$ & 7.3 & $68(6.0)$ & 3.6 & $236(8.5)$ & 7.2 & $68(6.0)$ & 5.9 \\
\hline$\geq 35$ years & $1061(1.7)$ & 6.7 & $21(1.9)$ & 2.6 & $80(2.9)$ & 16.3 & $21(1.9)$ & 4.8 \\
\hline Missing & $5881(9.4)$ & & $102(9.0)$ & & $371(13.3)$ & & $102(9.0)$ & \\
\hline \multicolumn{9}{|l|}{$\begin{array}{l}\text { Numbers of early termination of } \\
\text { pregnancy }\end{array}$} \\
\hline None & $41582(66.3)$ & 5.9 & $725(63.8)$ & 3.8 & $1940(69.8)$ & 5.9 & $725(63.8)$ & 5.0 \\
\hline One & $13687(21.8)$ & 5.7 & $244(21.5)$ & 4.6 & $559(20.1)$ & 5.6 & $244(21.5)$ & 3.7 \\
\hline Two or more & $7267(11.6)$ & 5.9 & $159(14.0)$ & 4.3 & $263(9.5)$ & 6.5 & $159(14.0)$ & 6.3 \\
\hline Missing & $181(0.3)$ & & $9(0.8)$ & & $19(0.7)$ & & $9(0.8)$ & \\
\hline \multicolumn{9}{|l|}{ Ever been pregnant } \\
\hline No & $4678(7.5)$ & 7.0 & $64(5.6)$ & 6.8 & $254(9.1)$ & 6.7 & $64(5.6)$ & 7.8 \\
\hline Yes & $57956(92.4)$ & 5.8 & $1073(94.4)$ & 4.0 & $2526(90.8)$ & 5.8 & $1073(94.4)$ & 4.8 \\
\hline Missing & $83(0.1)$ & & $0(0.0)$ & & $1(0.0)$ & & $0(0.0)$ & \\
\hline \multicolumn{9}{|l|}{ Age when first became pregnant $\mathrm{d}$} \\
\hline$\leq 19$ years & $13951(22.2)$ & 4.9 & $372(32.7)$ & 4.5 & $328(11.8)$ & 4.3 & $372(32.7)$ & 4.8 \\
\hline $20-24$ & $29676(47.3)$ & 5.7 & $468(41.2)$ & 3.3 & $1078(38.8)$ & 5.8 & 468 (41.2) & 4.9 \\
\hline $25-29$ & $11033(17.6)$ & 6.4 & $156(13.7)$ & 4.2 & $827(29.7)$ & 5.2 & $156(13.7)$ & 3.2 \\
\hline $30-34$ & $2456(3.9)$ & 6.9 & $52(4.6)$ & 3.2 & $206(7.4)$ & 8.3 & $52(4.6)$ & 7.7 \\
\hline$\geq 35$ & 767 (1.2) & 7.8 & $19(1.7)$ & 4.4 & $63(2.3)$ & 15.9 & $19(1.7)$ & 5.3 \\
\hline Missing & $4834(7.7)$ & & $70(6.2)$ & & $279(10.0)$ & & $70(6.2)$ & \\
\hline \multicolumn{9}{|l|}{ Number of pregnancies } \\
\hline None & $4678(7.5)$ & 7.0 & $222(5.4)$ & 6.8 & $254(9.1)$ & 6.7 & $64(5.6)$ & 7.8 \\
\hline One & $3417(5.5)$ & 6.4 & $370(9.0)$ & 3.8 & $185(6.7)$ & 6.0 & $64(5.6)$ & 3.1 \\
\hline Two & $11032(17.6)$ & 6.0 & $642(15.7)$ & 3.9 & $627(22.6)$ & 7.2 & $180(15.8)$ & 6.1 \\
\hline Three to four & $25650(40.9)$ & 5.8 & $1420(34.6)$ & 4.7 & $1158(41.6)$ & 5.6 & 424 (37.3) & 4.0 \\
\hline Five to nine & $16637(26.5)$ & 5.5 & $1281(31.3)$ & 3.3 & $516(18.6)$ & 4.7 & $366(32.2)$ & 5.2 \\
\hline More than ten & $1183(1.9)$ & 4.3 & 147 (3.6) & 4.8 & $24(0.9)$ & 8.3 & $37(3.3)$ & 5.4 \\
\hline Missing & $120(0.2)$ & & $17(0.4)$ & & $17(0.6)$ & & $2(0.2)$ & \\
\hline $\begin{array}{l}\text { Ever tried to become pregnant fo } \\
\text { a year or more }\end{array}$ & & & & & & & & \\
\hline No & $53302(85.0)$ & 5.7 & $1010(88.8)$ & 4.0 & $2330(83.8)$ & 5.9 & $1010(88.8)$ & 4.6 \\
\hline Yes & $9204(14.7)$ & 6.5 & $115(10.1)$ & 5.2 & $400(14.4)$ & 6.0 & $115(10.1)$ & 7.8 \\
\hline Missing & $211(0.3)$ & & $12(1.1)$ & & $51(1.8)$ & & $12(1.1)$ & \\
\hline Age at menarche & & & & & & & & \\
\hline$\leq 11$ years & $12578(20.1)$ & 6.2 & $256(22.5)$ & 4.7 & $539(19.4)$ & 6.1 & $256(22.5)$ & 5.1 \\
\hline $12-13$ years & 33951 (54.1) & 5.8 & $577(50.8)$ & 4.0 & 1377 (49.5) & 6.5 & $577(50.8)$ & 4.9 \\
\hline $14-15$ years & 13364 (21.3) & 5.7 & $238(20.9)$ & 4.2 & $625(22.5)$ & 5.4 & $238(20.9)$ & 4.6 \\
\hline$\geq 16$ years & $2680(4.3)$ & 5.7 & $58(5.1)$ & 5.3 & $210(7.6)$ & 2.9 & $58(5.1)$ & 6.9 \\
\hline Missing & $144(0.2)$ & & $8(0.7)$ & & $30(1.1)$ & & $8(0.7)$ & \\
\hline Age at menopause & & & & & & & & \\
\hline$<40$ & $8352(13.3)$ & 4.9 & $233(20.5)$ & 3.7 & $244(8.8)$ & 7.0 & $233(20.5)$ & 3.0 \\
\hline $40-44$ & $8770(14.0)$ & 5.4 & $181(15.9)$ & 4.9 & $315(11.3)$ & 3.8 & $181(15.9)$ & 5.0 \\
\hline $45-49$ & $14791(23.6)$ & 5.6 & $277(24.4)$ & 3.8 & $702(25.2)$ & 4.8 & $277(24.4)$ & 4.7 \\
\hline $50-54$ & 23125 (36.9) & 6.1 & $336(29.6)$ & 3.9 & $1190(42.8)$ & 7.1 & $336(29.6)$ & 6.3 \\
\hline$\geq 55$ & $7178(11.5)$ & 7.2 & $101(8.9)$ & 5.8 & $282(10.1)$ & 5.0 & $101(8.9)$ & 5.0 \\
\hline Missing & $501(0.8)$ & & $9(0.8)$ & & $48(1.7)$ & & $9(0.8)$ & \\
\hline Menopausal type ${ }^{b}$ & & & & & & & & \\
\hline Natural Menopause & $38914(62.1)$ & 6.0 & $606(53.3)$ & 4.6 & $2022(72.7)$ & 6.1 & $606(53.3)$ & 6.3 \\
\hline Radiation/Drug Therapy & $20374(32.5)$ & 5.3 & $494(43.5)$ & 3.6 & $634(22.8)$ & 5.5 & $494(43.5)$ & 3.2 \\
\hline Surgery & $2228(3.6)$ & 5.2 & $27(2.4)$ & 2.0 & $51(1.8)$ & 2.0 & $27(2.4)$ & 3.7 \\
\hline Missing & $1201(1.9)$ & & $10(0.9)$ & & $74(2.7)$ & & $10(0.9)$ & \\
\hline
\end{tabular}


Table 4. Associations between reproductive factors and breast cancer in PLCO participants stratified by races/ethnicities.

\begin{tabular}{|c|c|c|c|c|c|c|c|c|c|}
\hline \multirow[t]{2}{*}{ Reproductive factors } & \multicolumn{2}{|c|}{ non-Hispanic Caucasians } & \multicolumn{2}{|c|}{$\begin{array}{l}\text { non-Hispanic } \\
\text { African Americans }\end{array}$} & \multicolumn{2}{|c|}{$\begin{array}{l}\text { non-Hispanic Asian/Pacific } \\
\text { Islander }\end{array}$} & \multicolumn{2}{|l|}{ Hispanics } & \multirow[t]{2}{*}{$\begin{array}{l}\text { P value of } \\
\text { interactions }\end{array}$} \\
\hline & $\begin{array}{l}\text { Adjusted HRa } \\
(95 \% \text { CI })\end{array}$ & $P$-valueb & $\begin{array}{l}\text { Adjusted HRa } \\
(95 \% \text { CI })\end{array}$ & $P$-value ${ }^{b}$ & $\begin{array}{l}\text { Adjusted HRa } \\
(95 \% \text { CI })\end{array}$ & $P$-value ${ }^{b}$ & $\begin{array}{l}\text { Adjusted HRa } \\
(95 \% \mathrm{CI})\end{array}$ & $P$-value ${ }^{b}$ & \\
\hline Ever take oral contraceptive pills & & & & & & & & & 0.047 \\
\hline No & Ref & & Ref & & Ref & & Ref & & \\
\hline Yes & $1.09(1.01,1.18)$ & 0.037 & $0.95(0.64,1.42)$ & 0.814 & $0.93(0.63,1.39)$ & 0.725 & $0.88(0.45,1.71)$ & 0.698 & \\
\hline $\begin{array}{l}\text { Menopausal hormone therapy } \\
\text { use }\end{array}$ & & & & & & & & & 0.288 \\
\hline Never & Ref & & Ref & & Ref & & & & \\
\hline Former user & $1.08(0.96,1.21)$ & 0.226 & $0.76(0.46,1.25)$ & 0.278 & $1.55(0.90,2.66)$ & 0.112 & $0.73(0.25,2.13)$ & 0.569 & \\
\hline Current user less than 5 years & $1.24(1.10,1.39)$ & $<0.001$ & $1.04(0.59,1.85)$ & 0.895 & $1.25(0.71,2.22)$ & 0.443 & $1.15(0.48,2.78)$ & 0.755 & \\
\hline Current user more than 5 years & $1.44(1.31,1.59)$ & $<0.001$ & $1.04(0.61,1.78)$ & 0.880 & $1.98(1.23,3.20)$ & 0.005 & $0.80(0.35,1.85)$ & 0.608 & \\
\hline Age at birth of first child & & & & & & & & & 0.526 \\
\hline$\leq 19$ & Ref & & Ref & & Ref & & Ref & & \\
\hline $20-24$ & $0.88(0.73,1.05)$ & 0.147 & $0.80(0.41,1.55)$ & 0.505 & $1.57(0.40,6.11)$ & 0.517 & $0.38(0.08,1.72)$ & 0.207 & \\
\hline $25-29$ & $0.94(0.75,1.19)$ & 0.616 & $0.41(0.13,1.31)$ & 0.133 & $1.06(0.21,5.33)$ & 0.945 & $0.03(0.00,1.38)$ & 0.073 & \\
\hline $30-34$ & $1.16(0.85,1.57)$ & 0.347 & $0.60(0.15,2.48)$ & 0.482 & $0.92(0.14,6.02)$ & 0.932 & - & - & \\
\hline$\geq 35$ & $0.84(0.53,1.33)$ & 0.450 & $0.46(0.05,3.92)$ & 0.478 & $3.06(0.41,22.68)$ & 0.273 & - & - & \\
\hline Numbers of early termination of $p$ & pregnancy & & & & & & & & 0.391 \\
\hline None & Ref & & Ref & & Ref & & Ref & & \\
\hline Once & $1.02(0.93,1.12)$ & 0.736 & $1.26(0.82,1.93)$ & 0.298 & $1.21(0.76,1.91)$ & 0.426 & $0.72(0.29,1.77)$ & 0.469 & \\
\hline Twice or more & $1.08(0.95,1.23)$ & 0.220 & $1.20(0.72,2.00)$ & 0.489 & $1.91(0.99,3.68)$ & 0.054 & $1.37(0.53,3.52)$ & 0.516 & \\
\hline Age when first became pregnant & & & & & & & & & 0.946 \\
\hline$\leq 19$ & Ref & & Ref & & Ref & & Ref & & \\
\hline $20-24$ & $1.20(1.02,1.41)$ & 0.028 & $0.81(0.41,1.59)$ & 0.540 & $1.27(0.39,4.10)$ & 0.696 & $2.10(0.47,9.45)$ & 0.334 & \\
\hline $25-29$ & $1.18(0.94,1.48)$ & 0.154 & $1.32(0.40,4.35)$ & 0.647 & $1.63(0.38,7.03)$ & 0.516 & $\begin{array}{l}11.72(0.28 \\
493.33)\end{array}$ & 0.197 & \\
\hline $30-34$ & $1.09(0.78,1.51)$ & 0.624 & $0.58(0.09,3.95)$ & 0.577 & $2.26(0.38,13.48)$ & 0.370 & - & - & \\
\hline$\geq 35$ & $1.64(0.96,2.82)$ & 0.073 & $1.51(0.08,28.33)$ & 0.782 & $1.72(0.23,12.96)$ & 0.597 & - & - & \\
\hline Number of pregnancies & & & & & & & & & 0.951 \\
\hline None & Ref & & Ref & & Ref & & Ref & & \\
\hline One & - & & - & & & & & & \\
\hline Two & $0.92(0.77,1.09)$ & 0.321 & $0.95(0.42,2.17)$ & 0.905 & $1.69(0.74,3.87)$ & 0.212 & $1.67(0.30,9.17)$ & 0.556 & \\
\hline Three to four & $0.91(0.77,1.08)$ & 0.301 & $1.14(0.53,2.43)$ & 0.743 & $1.26(0.54,2.93)$ & 0.598 & $1.02(0.19,5.48)$ & 0.986 & \\
\hline Five to nine & $0.89(0.74,1.07)$ & 0.208 & $0.69(0.30,1.58)$ & 0.380 & $0.94(0.35,2.54)$ & 0.903 & $1.29(0.22,7.63)$ & 0.782 & \\
\hline More than ten & $0.68(0.48,0.96)$ & 0.028 & $1.04(0.34,3.20)$ & 0.952 & $0.70(0.08,6.52)$ & 0.755 & $1.76(0.17,18.48)$ & 0.638 & \\
\hline $\begin{array}{l}\text { Ever tried to become pregnant for } \\
\text { a year or more }\end{array}$ & & & & & & & & & 0.774 \\
\hline No & Ref & & Ref & & Ref & & Ref & & \\
\hline Yes & $1.08(0.97,1.20)$ & 0.146 & $1.02(0.53,1.94)$ & 0.962 & $0.91(0.55,1.52)$ & 0.714 & $2.60(1.05,6.46)$ & 0.039 & \\
\hline Age at menarche & & & & & & & & & 0.806 \\
\hline$\leq 11$ years & Ref & & Ref & & Ref & & Ref & & \\
\hline $12-13$ years & $0.92(0.83,1.00)$ & 0.057 & $0.78(0.51,1.20)$ & 0.265 & $1.28(0.78,2.09)$ & 0.330 & $0.68(0.32,1.48)$ & 0.336 & \\
\hline $14-15$ years & $0.91(0.81,1.01)$ & 0.088 & $0.87(0.53,1.45)$ & 0.599 & $1.22(0.69,2.17)$ & 0.498 & $0.68(0.27,1.71)$ & 0.410 & \\
\hline$\geq 16$ years & $0.88(0.73,1.07)$ & 0.209 & $0.83(0.36,1.91)$ & 0.658 & $0.53(0.18,1.57)$ & 0.252 & $1.09(0.29,4.16)$ & 0.898 & \\
\hline Age at menopause & & & & & & & & & 0.717 \\
\hline$<40$ & Ref & & Ref & & Ref & & Ref & & \\
\hline $40-44$ & $1.07(0.92,1.24)$ & 0.366 & $1.68(0.93,3.04)$ & 0.084 & $0.42(0.16,1.13)$ & 0.084 & $2.09(0.68,6.46)$ & 0.198 & \\
\hline $45-49$ & $1.06(0.92,1.23)$ & 0.394 & $1.07(0.57,2.00)$ & 0.835 & $0.63(0.27,1.44)$ & 0.269 & $1.96(0.61,6.34)$ & 0.260 & \\
\hline $50-54$ & $1.15(0.99,1.33)$ & 0.060 & $0.83(0.42,1.61)$ & 0.573 & $1.07(0.47,2.44)$ & 0.880 & $1.44(0.43,4.78)$ & 0.553 & \\
\hline$\geq 55$ & $1.25(1.06,1.49)$ & 0.009 & $1.19(0.54,2.61)$ & 0.668 & $0.73(0.27,1.98)$ & 0.531 & $0.71(0.12,4.33)$ & 0.706 & \\
\hline Menopausal type & & & & & & & & & 0.026 \\
\hline Natural Menopause & Ref & & Ref & & Ref & & Ref & & \\
\hline Radiation/Drug Therapy & $1.17(0.99,1.39)$ & 0.075 & $0.40(0.05,2.98)$ & 0.369 & $0.37(0.05,2.69)$ & 0.322 & $1.55(0.18,13.29)$ & 0.692 & \\
\hline Surgery & $0.88(0.79,0.98)$ & 0.016 & $0.62(0.38,1.01)$ & 0.053 & $0.80(0.44,1.46)$ & 0.464 & $0.76(0.31,1.85)$ & 0.546 & \\
\hline
\end{tabular}

A novel finding of this study was that advanced age at natural menopause was associated with increased risk of breast cancer only in non-Hispanic Caucasians but not in non-Hispanic African Americans, non-Hispanic Asian/Pacific Islanders, and Hispanics. We suspected that this phenomenon could be due to the unequal distributions of types of breast cancer across races/ethnicities. Our supplementary data analysis showed that about $13 \%$ of breast cancer patients in non-Hispanic Caucasian group were lobular or tubular types, where as in African American, non-Hispanic Asian/Pacific Islander, and Hispanic groups, lobular or tubular types accounted for $11 \%, 6 \%$, and $10 \%$, respectively. Compared with ductal type, lobular or tubular types of breast cancer are more tightly associated with 
estrogen levels ${ }^{24}$. In addition, breast cancer related gene polymorphisms may also mediate the lower breast cancer risk in non-Hispanic African Americans, non-Hispanic Asian/Pacific Islanders, and Hispanics, since previous study indicated that the spectrum of BRCA1/BRCA2 differed across races/ethnicities ${ }^{25}$.

The PLCO dataset provide us a unique opportunity to examine the association between extremly high number of pregnancies and breast cancer by races/ethnicities. We found that only non-Hispanic Caucasian group with 10 or more times of pregnancies had reduced breast cancer risk, which indicated that pregnancy might need multiple episodes to play its breast cancer protection effect. Consistent with previous studies ${ }^{26,27}$, we found that surgery induced menopause was associated with reduced risk of breast cancer in non-Hispanic Caucasians, which can be explained by the shortened duration of estrogen exposure time. Although bilateral prophylactic salpingo-oophorectomy could significantly reduce woman's breast cancer risk, cautious is needed to take when considering using risk-reducing surgeries, as surgical menopause could cause abrupt onset of menopausal symptoms and lead to lifelong side effects such as osteoporosis and vaginal dryness. Women who have severe menopausal symptoms after surgery might seek for MHT which is a well-established risk factor for breast cancer. We speculate that the reduced breast cancer effect by surgery might be attenuated to null by following MHT use.

Some studies but not all have suggested that early termination of pregnancy was associated with increased risk of breast cancer ${ }^{28}$. They believed that early termination of pregnancy, which often occurs at the first trimester of pregnancy when breast cells were undergoing great proliferation, was an abrupt interruption of pregnancy ${ }^{29}$. As a result, the breast will contain a high number of undifferentiated cells, which may influence woman's subsequent breast cancer risk. In this study, we observed that 2 or more times of early termination of pregnancy was associated with borderline increased risk of breast cancer only among non-Hispanic Asian/Pacific Islanders but not in other race groups, informing the association between early termination of pregnancy and breast cancer risk is weak.

This study had several limitations. First, the relative small sample sizes of minority race groups might impact the robustness of our findings. Some of the differences between race groups could be due to chance only, as most of our interaction analysis were non-significant. Replications are needed in larger samples. Second, our analytic sample was embedded in PLCO study which was a randomized clinical trial and not population based. Thus the participants in each race group might be not a representative sample of that race. Third, we were unable to control important genetic factors in this study and therefore cannot know the extent to which our findings may be affected by these factors. Fourth, self-reported reproductive factors especially some of the sensitive reproductive factors (e.g., abortion) may be subjected to recall bias but this is unlikely given the risk factor data was collected prior to cancer diagnosis. Fifth, due to the small numbers of breast cancer patients in non-Hispanic Caucasian ethnic groups, we didn't further explore the associations between reproductive factors and breast cancer among each race group by estrogen receptor status.

In conclusions, we found that different races/ethnicities had different breast cancer related reproductive risk factors. Non-Hispanic Caucasians had the most while other races/ethnicities only had few breast cancer related productive factors. Non-Hispanic Caucasians and non-Hispanic Asian/Pacific Islanders were at increased risk of developing breast cancer after MHT for more than five years. The effects of the reproductive factors on breast cancer were very similar among the minority race/ethnic groups, although most of the socio-demographic characteristics were different across the minority race/ethnic groups.

\section{Acknowledgment}

The authors thank the National Cancer Institute for access to NCI' $\mathrm{s}$ data collected by the Prostate, Lung, Colorectal and Ovarian (PLCO) Cancer Screening Trial. The statements contained herein are solely those of the authors and do not represent or imply concurrence or endorsement by NCI.

\section{Competing Interests}

The authors have declared that no competing interest exists.

\section{References}

1. Smigal C, Jemal A, Ward E, et al. Trends in breast cancer by race and ethnicity: update 2006. CA Cancer J Clin. 2006;56:168-183.

2. Ryerson $\mathrm{AB}$, Eheman $\mathrm{CR}$, Altekruse $\mathrm{SF}$, et al. Annual Report to the Nation on the Status of Cancer, 1975-2012, featuring the increasing incidence of liver cancer. Cancer. 2016;122:1312-1337.

3. Vainshtein J. Disparities in breast cancer incidence across racial/ethnic strata and socioeconomic status: a systematic review. Journal of the National Medical Association. 2008;100:833-839.

4. Bernstein L, Teal CR, Joslyn S, et al. Ethnicity-related variation in breast cancer risk factors. Cancer. 2003;97:222-229.

5. Lambertini M, Santoro L, Del ML, et al. Reproductive behaviors and risk of developing breast cancer according to tumor subtype: A systematic review and meta-analysis of epidemiological studies. Cancer Treatment Reviews. 2016;49:65-76.

6. Key TJ, Pike MC. The role of oestrogens and progestagens in the epidemiology and prevention of breast cancer. Eur J Cancer Clin Oncol. 1988;24:29-43.

7. Gail MH, Brinton LA, Byar DP, et al. Projecting individualized probabilities of developing breast cancer for white females who are being examined annually. J Natl Cancer Inst. 1989;81:1879-1886. 
8. Li R, Gilliland FD, Baumgartner K, et al. Hormone replacement therapy and breast carcinoma risk in Hispanic and non-Hispanic women. Cancer. 2002;95:960-968.

9. Gilliland FD, Hunt WC, Baumgartner KB, et al. Reproductive risk factors for breast cancer in Hispanic and non-Hispanic white women: the New Mexico Women's Health Study. American Journal of Epidemiology. 1998;148:683-692.

10. Hall IJ, Moorman PG, Millikan RC, et al. Comparative analysis of breast cancer risk factors among African-American women and White women. American Journal of Epidemiology. 2005;161:40-51.

11. Lee SJ, Kumashiro K. A report on the status of Asian Americans and Pacific Islanders in education: Beyond the "model minority" stereotype. Washington, DC: National Education Association. 2005

12. Gohagan JK, Prorok PC, Hayes RB, et al. The Prostate, Lung, Colorectal and Ovarian (PLCO) Cancer Screening Trial of the National Cancer Institute: history, organization, and status. Control Clin Trials. 2000;21:251S-272S.

13. Zhu H, Lei X, Feng J, et al. Oral contraceptive use and risk of breast cancer: a meta-analysis of prospective cohort studies. Eur J Contracept Reprod Health Care. 2012;17:402-414.

14. Isaksson E, von Schoultz E, Odlind V, et al. Effects of oral contraceptives on breast epithelial proliferation. Breast Cancer Res Treat. 2001;65:163-169.

15. Bernstein L, Teal CR, Joslyn S, et al. Ethnicity-related variation in breast cancer risk factors. Cancer. 2003;97:222-229.

16. Dawson DA. Trends in use of oral contraceptives--data from the 1987 National Health Interview Survey. Fam Plann Perspect. 1990;22:169-172.

17. Pachman DR, Jones JM, Loprinzi CL. Management of menopause-associated vasomotor symptoms: Current treatment options, challenges and future directions. Int J Womens Health. 2010;2:123-135.

18. Rossouw JE, Anderson GL, Prentice RL, et al. Risks and benefits of estrogen plus progestin in healthy postmenopausal women: principal results From the Women's Health Initiative randomized controlled trial. JAMA. 2002;288:321-333.

19. Hou N, Hong S, Wang W, et al. Hormone replacement therapy and breast cancer: heterogeneous risks by race, weight, and breast density. J Natl Cancer Inst. 2013;105:1365-1372.

20. Del CM, Hughes KS, Halpern E, et al. Racial differences in mammographic breast density. Cancer. 2003;98:590-596.

21. Brown AF, Perez-Stable EJ, Whitaker EE, et al. Ethnic differences in hormone replacement prescribing patterns. Journal of General Internal Medicine. 1999;14:663-669.

22. Chlebowski RT, Kuller LH, Prentice RL, et al. Breast cancer after use of estrogen plus progestin in postmenopausal women. $\mathrm{N}$ Engl J Med. 2009;360:573-587.

23. Visvanathan K, Yager JD. Ethnic Variations in Estrogen and Its Metabolites: Sufficient to Explain Differences in Breast Cancer Incidence Rates? J Natl Cancer Inst. 2016;108.

24. Kotsopoulos J, Chen WY, Gates MA, et al. Risk factors for ductal and lobular breast cancer: results from the nurses' health study. Breast Cancer Research. 2010;12:R106.

25. Kurian AW. BRCA1 and BRCA2 mutations across race and ethnicity: distribution and clinical implications. Curr Opin Obstet Gynecol. 2010;22:72-78.

26. Guillem JG, Wood WC, Moley JF, et al. ASCO/SSO review of current role of risk-reducing surgery in common hereditary cancer syndromes. Journal of Clinical Oncology. 2006;24:4642-4660.

27. Kauff ND, Domchek SM, Friebel TM, et al. Risk-reducing salpingo-oophorectomy for the prevention of BRCA1- and BRCA2-associated breast and gynecologic cancer: a multicenter, prospective study. Journal of Clinical Oncology. 2008;26:1331-1337.

28. Guo J, Huang Y, Yang L, et al. Association between abortion and breast cancer: an updated systematic review and meta-analysis based on prospective studies. Cancer Causes Control. 2015;26:811-819.

29. Daling JR, Malone KE, Voigt LF, et al. Risk of breast cancer among young women: relationship to induced abortion. Journal of the National Cancer Institute. 1994;86:1584-1592. 